\title{
ISOTOPIC COMPOSITION OF BIOSULFUR IN ENVIRONMENTAL SAMPLES
}

\author{
YOSHIKO ARIKAWA, YUKIKO IMAIZUMI, and AKIRA SASAKI ${ }^{+}$
}

Chemistry Department, Japan Women's University, Bunkyo-ku, Tokyo 112, Japan +Geological Laboratory, Faculty of Education, Ibaraki University, Mito 310, Japan

\begin{abstract}
Sulfur isotopic composition of biological samples has been determined to investigate the behaviors of sulfur in the environment. $\delta^{34} \mathrm{~S}$ values of fishes range from +17 to $20 \mathrm{o} / 00$ and show that sulfur comes from the sea-water salfate whose $\delta$ $\mathrm{S}$ value is $20 \mathrm{o} / \mathrm{oo}$. In contrast, $\delta^{34} \mathrm{~S}$ values of plants whose sulfur is derived from water soluble sulfate in soil and sulfur dioxide in the atmosphere are scattered in the wide range from -7 to $+14 \mathrm{o} / 00$. It became obvious that plants growing in the north hemisphere tend to show a less $\delta^{34} \mathrm{~S}$ value than those of south hemisphere. Meat sulfur coming from soil through the foodchain shows almost the same tendency as plant sulfur. These results are reflected to the $\delta^{34} \mathrm{~S}$ values for nail sulfur of humanbeing that stands on the top of the foodchain.
\end{abstract}

Key words sulfur isotopes, human sulfur, plant sulfur, food sulfur

Sulfur isotopic works for minerals, rocks and other geological samples have brought many informations on geological behavior of sulfur for these 50 years. Though sulfur is also an important element in the biosphere, systematic works have not done for the isotopic composition of biosulfur. Several works of the sulfur isotopic composition in biological samples were reported for human hair by Szabo et al (1), for kidney stones by Krouse et al $(2,3)$, for leaves by Nakai (4), and recently for human nails by Sasaki $(5,6)$. In this paper we will present the sulfur isotopic composition of some biological samples determined by our own methods $(7,8)$ and discuss the sulfur cycle in the environment.

\section{EXPERIMENTAL}

Apparatus A Parr 1108 oxygen combustion bomb was employed for the decomposition of samples. Isotopic measurement for sulfur dioxide was made with a Hitachi RMU-6 mass spectometer remodelled for isotopic ratio measurement and a Varian-MAT 250 mass spectrometer. A secondary ion mass spectrometer, Hitachi IMA-3A, was used for solid sample, silver sulfide, using $\mathrm{Cs}(+)$ ion as a primary ion.

Reagents Tin(II)-strong phosphoric acid and a zinc acetate absorbing solution were prepared by the procedure described in previous papers $(7,8)$.

Precedure The precise methods were written in previous papers $(7,8)$. Less than $1 \mathrm{~g}$ of biological sample is ignited in the Parr 1108 oxygen combustion bomb filled with oxygen to 30 atm and a little amount of water. The sulfur trioxide formed dissolves in water as sulfate ion to which hydrochloric acid and barium chloride solution are added to form barium sulfate. The dried barium salfate is heated with tin(II)-strong phosphoric acid at $280^{\circ} \mathrm{C}$ for $20 \mathrm{~min}$ under a flow of nitrogen. The hydrogen sulfide formed is absorbed in a zinc acetate solution to fix as the zinc sulfide which is inturn converted to silver sulfide by adding silver nitrate solution. Sulfur isotopic measurement is carried out by the mass spectrometer as sulfur dioxide or by the secondary ion mass spectrometer as silver sulfide as shown in Fig.1. In the former method the silver sulfide is burned with copper(II) oxide at $850^{\circ} \mathrm{C}$ to form sulfur dioxide which is further purified by a successive vacuum distillation. In the latter method silver sulfide is grounded and pressed into a thin film. Cs(+) ion is used as a primary ion and the emitted ${ }^{32} \mathrm{~S}(-)$ and ${ }^{34} \mathrm{~S}(-)$ secondary ions are measured. The isotopic ratio is expressed in terms of $\delta^{34} \mathrm{~S}$ permil value defined as $\delta^{34} \mathrm{~S} 0 / 00=(\mathrm{Rx} / \mathrm{Rsd}-1) \times 1000$, where $R$ means the ratio of ${ }^{34} S /{ }^{32} S$, and the subscripts $x$ and sd denote a sample of interest and the standard sample, respectively. The standard sample is Canyon Diablo troilite which is accepted as an international standard for sulfur isotopic measurement. 


\section{RESULTS AND DISCUSSION}

Isotopic composition of sulfur in foods and human nails are measured and results are shown in Figs.2 and 3. For seafoods their $\delta^{34} \mathrm{~S}$ values fall in the narrow range from +17 to 20 $0 / 00$, which means sulfur comes from sea water sulfate whose $\delta^{34} S$ value is 20 o/oo all over the sea. Cereals, potatoes, beans, vegetables, fruits and meats show variant $\delta^{34} S$ values from -7 to $+14 \mathrm{o} / 00$. Sulfur in plants is derived mainly from soil and partly from the atmosphere. Recently, in addition to the natural sources of sulfur, i.e. sea spray, volcanic gas, products by biochemical processes and so on, serious amount of anthropogenic sulfur, mainly in the form of sulfur dioxide from fossil fuels, is released into the atmosphere. Sulfur dioxide is oxidized and dissolved in rain water as sulfate ion which is taken by plants through leaves and roots. Therefore, the isotopic composition of sulfur in plants seems to be complicated. Meat sulfur shows the same tendency of $\delta^{34} \mathrm{~S}$ values as plant sulfur. As shown in Figs.2 and $3, \delta^{34} \mathrm{~S}$ values of human sulfur extracted from nails are correlative with those of plant sulfur. In both cases $\delta^{34} \mathrm{~S}$ values are characterized by the light values in North America, while those in South America, India and Oceania tend to show heavier values. Kusakabe et al (9) showed isotopic ratio of sulfur extracted from plants, soil and related materials in New Zealand, $\delta^{34} \mathrm{~S}$ values of which are generally heavy and especially $\delta^{34} \mathrm{~S}$ value of sulfate is close to that of the sea water sulfate. It seems that sea spray contributes to the sulfur source in soil. Even a rough estimate of isotopic composition of sulfur shows that $\delta^{34} \mathrm{~S}$ values tend to be lighter in the north hemisphere, two third of which is occupied by the land, than in the south hemisphere, two third of which is occupied by the sea. Therefore, in the south hemisphere sulfur in the environment may be attributed mainly to the sea water sulfate as seen in New Zealand. In the north hemisphere anthropogenic sulfur from industry may participate in the sulfur cycle. Generally $\delta^{34} \mathrm{~S}$ value of anthropogenic sulfur is lighter than that of sea water sulfate. Light $\delta^{34} \mathrm{~S}$ values of plants and human nails in North America seem to be affected by the anthropogenic sulfur.

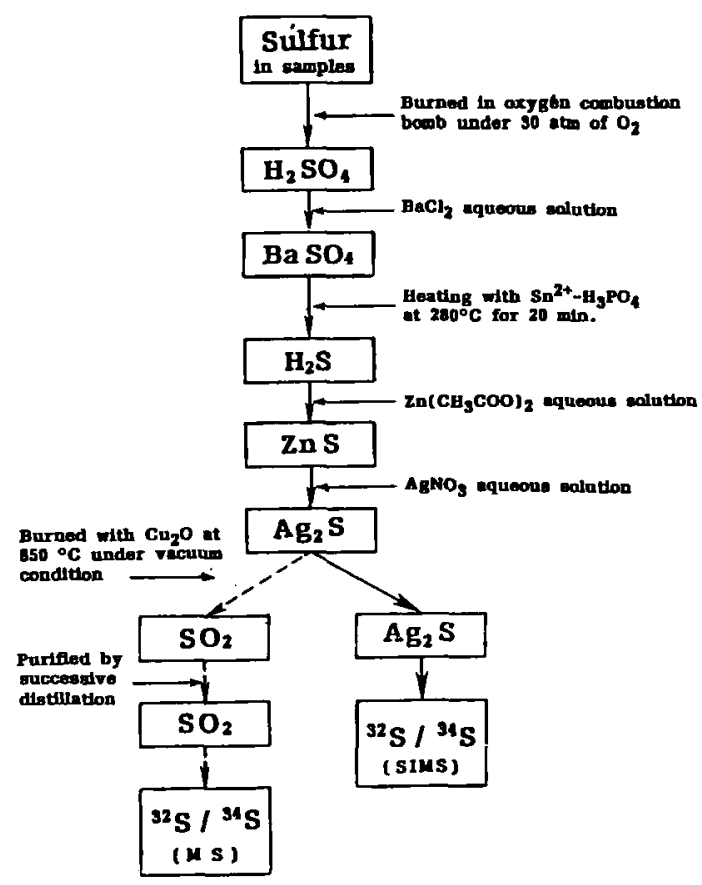

Fig.1: Flow chart for procedure.

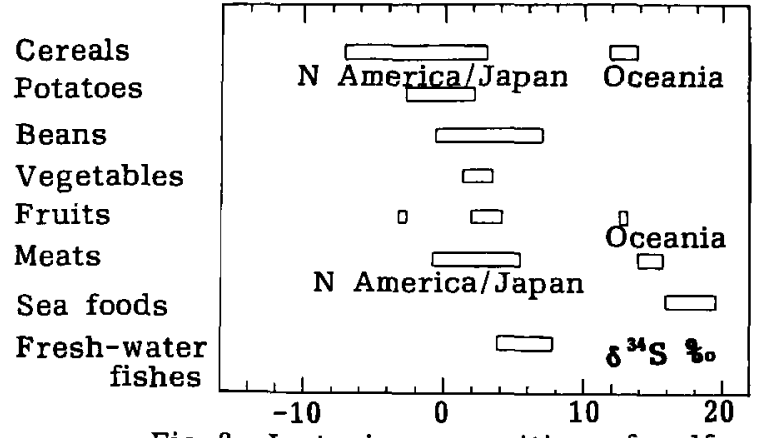

Fig. 2: Isotopic composition of sulfur in foods.

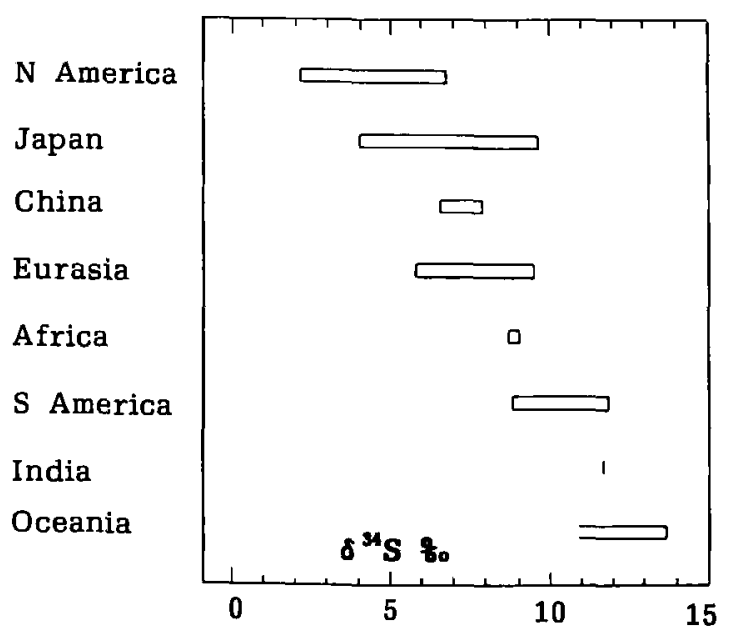

Fig. 3: Isotopic composition of sulfur in human nails.

\section{REFERENCES}

1. A. Szbo, A. Tudge, J. Macnmara and H. G. Thode, Science, 111, 464 (1950).

2. H. R. Krouse and A.A. Levinson, Geochim. Cosmochim. Acta, 48, 187 (1984).

3. H. R. Krouse, A. A. Levinson, D. Piggott and A. Ueda, App. Geochem., 2, 205 (1987).

4. N. Nakai, Gendaikagaku, 1984, 39 (1984).

5. A. Sasaki, J. Ibaraki Univ. (Natural Science), 39, 9 (1990).

6. A. Sasaki, Chishitu News, 444, 8 (1991).

7. Y. Arikawa and A. Sasaki, Anal. Sci., 3, 157 (1987).

8. Y. Arikawa, Y. Imaizumi and A. Sasaki, Nippon Kagaku Kaishi, 1991, 399 (1991).

9. M. Kusakabe, T. A. Rafter, J. D. Stout and T. W. Collie, New Zealand J. Sci., 19, 433 $(1976)$. 